\title{
Background retinopathy
}

National Diabetes Information Clearinghouse (NDIC)

\section{Definitions}

Diabetic retinopathy

Defined by National Diabetes Information Clearinghouse (NDIC)

Retina

Defined by National Diabetes Information Clearinghouse (NDIC)

Blood vessels

Defined by National Diabetes Information Clearinghouse (NDIC)

\section{Source}

National Diabetes Information Clearinghouse (U.S.). (2009). The diabetes dictionary. [Bethesda, Md.]: U.S. Dept. of Health and Human Services, National Institutes of Health, National Institute of Diabetes and Digestive and Kidney Diseases, National Diabetes Information Clearinghouse.

A type of damage to the retina of the eye marked by bleeding, fluid accumulation, and abnormal dilation of the blood vessels. Background retinopathy is an early stage of diabetic retinopathy. Also called simple or nonproliferative retinopathy. 